\title{
ORIGINAL ARTICLE \\ Characterization of unexpected postural changes during robot-assisted gait training in paraplegic patients
}

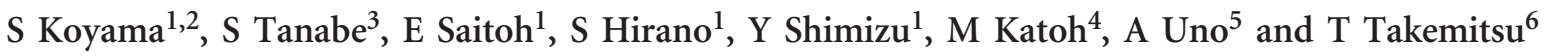

Study design: This is a retrospective study.

Objectives: The objectives of this study were to categorize unexpected postural changes (UPCs) during gait training in paraplegic patients with wearable gait-assist robots, to reveal the incidence of the UPC and its time-dependent changes during initial gait training period and to investigate neurological level-specific differences.

Setting: This study was conducted in Fujita Health University, Aichi, Japan.

Methods: We investigated five patients (46.2 \pm 14.6 years; lesion level: T6:3, T12:2). All patients had previously achieved gait with wearable robot and walker at supervision level. The UPCs were counted for 2 years and classified according to their type. The time-course data were calculated from the incidence of UPCs for 10 days from initial gait training with the walker. The neurological level-specific differences were investigated between T6 and T12 injuries.

Results: Eighty-five UPCs were observed and classified into three categories: anterior breakdown, posterior breakdown (PBD) and mal-timing. The average rate over the entire period was $0.96 \pm 0.62$ (incidents/h/subject). PBD, which was defined as hyperflexion of both hip joints, occurred with the highest frequency $(0.64 \pm 0.64$ incidents/h/subject). During initial gait training, there was a gradual decrease in the occurrence of UPC. For neurological level-specific differences, UPCs were observed more frequently in T6 injuries ( $1.36 \pm 0.35$ incidents/h/subject) compared with T12 injuries $(0.36 \pm 0.31$ incidents/h/subject).

Conclusion: PBDs might be the result of near collisions between the trunk of the user and the walker, which make it difficult for the users to move their trunk over an anterior stance limb. Training that is focused upon well-timed forward movements of the walker might be required to avoid the occurrence of this common UPC.

Spinal Cord (2016) 54, 120-125; doi:10.1038/sc.2015.138; published online 11 August 2015

\section{INTRODUCTION}

Complete motor spinal cord injury (SCI) patients (American Spinal Injury Association classification $\mathrm{A}$ or $\mathrm{B})^{1}$ lose voluntary neural control of the limbs below the level of the lesion. Many of these patients cannot achieve independent standing or are unable to walk after rehabilitation. ${ }^{2}$ Morganti et al. ${ }^{3}$ reported that $\sim 90 \%$ of complete SCI patients are dependent on a wheelchair. The long-term use of a wheelchair causes medical problems ${ }^{4}$ and psychosocial problems as the result of a relatively low eye level., ${ }^{5,6}$ Thus, gait reconstruction in such patients has been a key issue in rehabilitation medicine.

To achieve gait reconstruction, orthoses are available. ${ }^{7,8}$ Orthoses are classified as the bilateral external joint type (lateral system) and the medial single hip joint type (medial system), according to the position of the orthotic hip joint. As compared with the lateral system, the medial system, for example, Primewalk, has excellent standing stability and wheelchair compatibility. ${ }^{8}$ However, for progression and lateral motion of the body during walking with an orthosis, the upper extremities have to exert power. The high loads on the upper limbs during gait may be a cause of the high rate of energy expenditure in such patients. ${ }^{9}$
To solve these problems of gait reconstruction with an orthosis in SCI patients, a newly wearable robot for gait assistance, the so-called Wearable Power-Assist Locomotor (WPAL), for gait reconstruction in SCI patients has been proposed in the clinical setting. ${ }^{10,11}$ WPAL is characterized by a single medial hip joint located between both thighs and has no pelvic or trunk support, similar to Primewalk. Standing stability in SCI patients is obtained by consistently maintaining the C-posture, which means that the knee and ankle joints are locked in the full extension and intermediate positions, respectively (Figure 1). During gait with WPAL, SCI patients need to maintain the C-posture and move in the forward and mediolateral direction in accordance with the walk assistance timing of WPAL using a walker. ${ }^{10,11}$ Simultaneously, the patients are required to move the walker forward at the correct timing.

Because of the insufficient acquisition of coordination between the weight shift of the SCI patient and the leg movements of the WPAL, unexpected postural changes (UPCs) might occur during the initial phase of WPAL training. For WPAL users, complete falling episodes with dangerous condition do not occur because the walker provides a movable external support that reduces the risk of a rapid fall.

${ }^{1}$ Department of Rehabilitation Medicine I, School of Medicine, Fujita Health University, Toyoake, Japan; ${ }^{2}$ Department of Rehabilitation, Kawamura Hospital, Gifu, Japan; ${ }^{3}$ Faculty of Rehabilitation, School of Health Sciences, Fujita Health University, Toyoake, Japan; ${ }^{4}$ Department of Rehabilitation, Fujita Health University Hospital, Toyoake, Japan; ${ }^{5}$ Tomei Brace Company, Seto, Japan and ${ }^{6}$ ASKA Corporation, Kariya, Japan

Correspondence: Dr S Tanabe, Department of Rehabilitation Medicine I, School of Medicine, Fujita Health University, 98-1 Dengakugakubo, Kutsukake, Toyoake 470-1192, Aichi, Japan.

E-mail: tanabes@fujita-hu.ac.jp

Received 9 February 2015; revised 3 May 2015; accepted 22 June 2015; published online 11 August 2015 


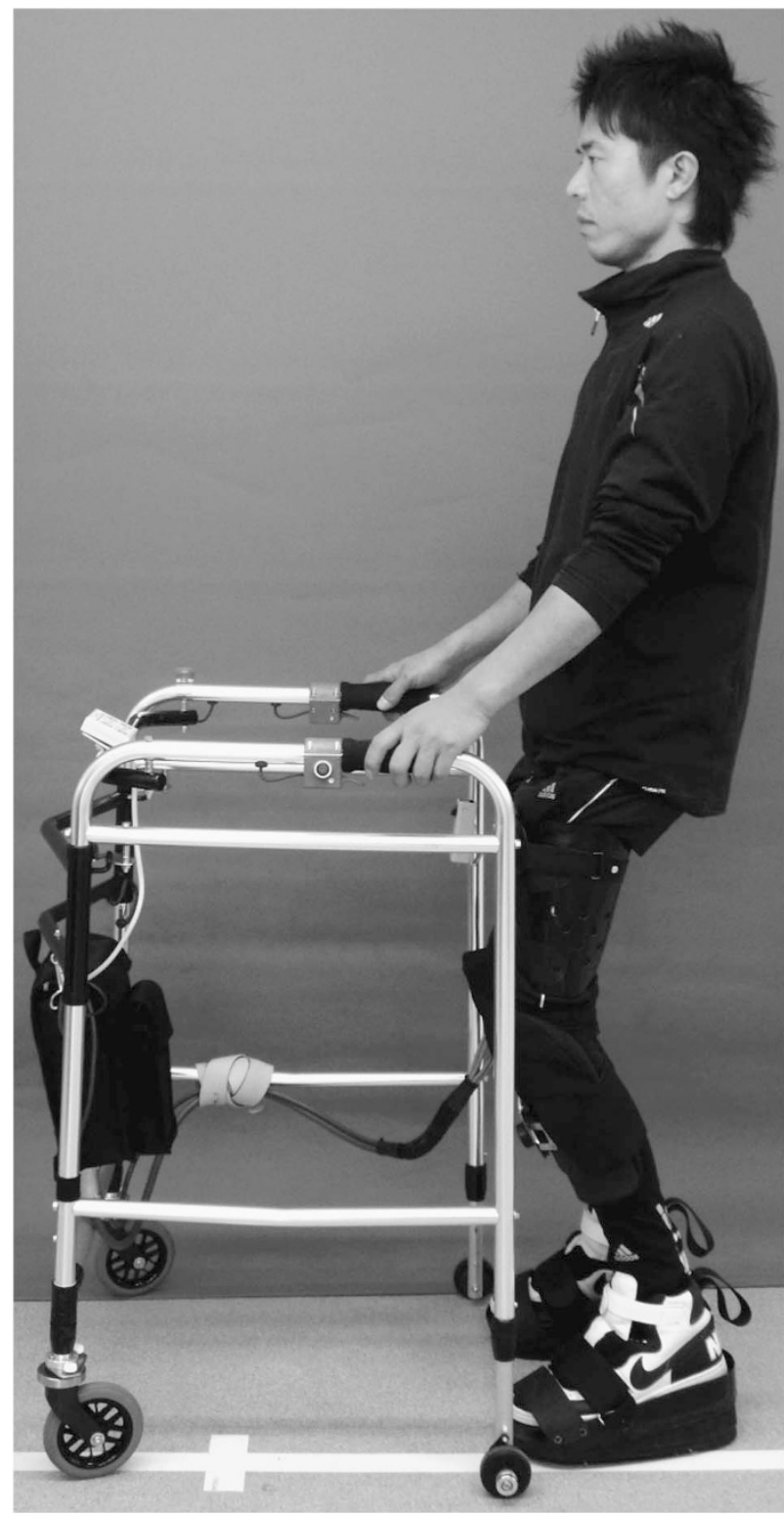

Figure 1 Upright position with the C-posture (subject E).

However, knowledge of the occurrence of UPCs during gait training with a wearable gait-assist robot would facilitate the development of a more effective training program for SCI patients with such a device.

The aims of this study were to classify UPCs, to reveal the incidence of each type of UPCs during gait training and its time-dependent changes during the initial gait training period and to investigate the relationship between the frequency of UPCs and the neurological level.

\section{MATERIALS AND METHODS}

\section{Subjects}

The subjects of this study were five patients with complete SCI (four males and one female, $46.2 \pm 14.6$ years old). Their clinical characteristics are shown in Table 1. All patients had an American Spinal Injury Association classification of A or B during the final stages of their rehabilitation program. The patients did not have lesions of the upper extremities, nor orthostatic hypotension in the upright position. They had achieved independent activities of daily living with wheelchair. In the investigation of the relationship between the frequency of
Table 1 Patient demographics

\begin{tabular}{lccclc}
\hline Subject & Age (years) & Gender & After onset (years) & Lesion level & ASIA \\
\hline A & 62 & M & 9 & Th6 & A \\
B & 43 & M & 9 & Th6 & A \\
C & 61 & F & 16 & Th6 & A \\
D & 33 & M & 16 & Th12 & A \\
E & 32 & M & 6 & Th12 & B \\
\hline
\end{tabular}

Abbreviation: ASIA, American Spinal Injury Association classification.

UPCs and the level of injury, the patients were divided into a high-lesion group (T6 lesions) and a low-lesion group (T12 lesions). Each subject was informed of the purpose of this study and gave informed consent to participate in the study. This study followed the Declaration of Helsinki Principles and was conducted after receiving approval from the ethics committee of Fujita Health University.

\section{Wearable Power-Assist Locomotor}

As stated above, WPAL is characterized as a medial system. The robotic part permits movements with six degrees-of-freedom. Each joint has an individual actuator, and thus the hip, knee and ankle joints perform flexion-extension movements independently. WPAL is used with a custom walker. ${ }^{10,11}$

WPAL requires users to synchronize rhythmic lateral weight shifts with the robot's motion, which can be learned with a staged gait training approach. ${ }^{10,11}$ In the first stage, users practice stepping with the WPAL. The second stage is gait exercise using parallel bars. The third stage is gait exercise on a treadmill. During these three stages, patients use a safety harness; when UPCs occur, falls are prevented completely by the harness. The final stage is gait exercise using the walker. At the beginning of the final stage, the patients walk under a suspension system using the walker. However, when their gait becomes stable and safe, they walk freely around the training room without the safety harness. UPCs that occur without the safety harness during this phase can lead to a fall. Hence, the final stage without the harness is the most important period for the prevention of UPCs from the perspective of system design. All patients in the present study had just progressed to the final stage without the harness before they were observed.

\section{Data collection and analysis}

For 2 years, data were collected at the University Hospital. Each trial of gait training with WPAL lasted $90 \mathrm{~min}$. Approximately, 60-70 min were spent in the gait trial; the remaining time was spent checking the physical condition of the subjects. Trials were performed in a rehabilitation training room with an even-surface floor and dimensions of $10 \mathrm{~m}$ on a side. During the walking trials, a physical therapist observed each subject from behind. The physical therapist did not intervene unless the subject was unable to recover from a UPC. If the subject was unable to self-recover with or without pushing the stop button, the physical therapist assisted with recovery. The gait trials with WPAL were recorded using a digital video camera (HDR-AX2000; Sony, Tokyo, Japan).

The number of UPCs was counted by checking the records of the video retrospectively. The criteria of UPC was that users could not keep continuous and steady walking with WPAL. In other words, if users could continue steady walking, all postural sways were judged as the normal ranges. Then, UPCs were classified into several categories. The total incidence of UPCs was calculated; then, time-course data were calculated from the incidence for 10 days from initial gait training with the walker without the harness. The change of frequency of UPCs with the progress of gait training was assessed using regression analysis (correlation between trial numbers versus frequency of UPCs). The Shapiro-Wilk test was used to determine whether variables were normally distributed. With normally distributed responses, Pearson's correlation coefficient was used. In contrast, if the data were not normally distributed, Spearman's rank correlation coefficient was used. All statistical analyses were performed with R (2.8.1; Institute of Statistical Mathematics, Tokyo, Japan). Statistical significance was set at $P<0.05$. In addition, the observed UPCs were separated into neurological level. 


\section{RESULTS}

The total trial time was $121.5 \mathrm{~h}$. The time required to check the physical condition and for standing/sitting scenes was excluded from the observations. As a result, $86 \mathrm{~h}$ of walking time was used in the present study. UPCs were observed 85 times. There was no observation of a nonrecoverable fall during gait with WPAL. If UPC was moderate, patients could self-recover with WPAL motion on. On the other hand, patients could self-recover by pushing the stop button to stop WPAL motion when a severe UPC occurred.

The UPCs were classified into three types. The first type was an anterior breakdown (ABD): both hip joints become hyperextended compared with the hip extension angle of ordinary walking pattern with WPAL, because of the long distance between the subject and the walker during gait, and cause a forward change of walking posture. The second type was defined as a posterior breakdown (PBD): both hip joints become hyperflexed compared with the hip flexion angle of ordinary walking pattern with WPAL, because of the short distance between the subject and the walker during gait, and cause a backward change of walking posture. The third type was defined as mal-timing (MT): the timing of weight shift in the subjects was different from the timing of the gait cycle of the WPAL, which caused a lateral change of walking posture (Figure 2).
In the incidence of UPCs, the average rate over the entire period was $0.96 \pm 0.62$ (incidents/h/subject; Table 2). With regard to the classification of UPCs, PBDs occurred most frequently $(0.64 \pm 0.64$ incidents/h/subject), followed by MTs $(0.20 \pm 0.09$ incidents/h/subject $)$ and ABDs $(0.13 \pm 0.14$ incidents/h/subject; Table 3). During the 10 days of initial gait training, the time-course data of all types of UPC showed a gradual decrease in their incidence (Figure 3). With the progress of gait training, the frequency of $\mathrm{PBD}$ was significantly decreased (Pearson's correlation coefficient $r=-0.7, P<0.05$ ). On the other hand, there was decreasing trend in the frequency of $\mathrm{ABD}$ and MT, but not significantly (Spearman's rank correlation coefficients $r=-0.3, P>0.05$; Pearson's correlation coefficient $r=-0.3, P>0.05$ ).

There was a difference between T6 and T12 injuries with respect to the frequency of UPCs. Seventy-two UPCs were observed in subjects with T6 injuries $(1.36 \pm 0.35$ incidents/h/subject; Table 2$)$, which account for $84.7 \%$ of the total number of UPCs. In contrast, only 13 UPCs were observed in subjects with T12 level injuries $(0.36 \pm 0.31$ incidents/h/subject; Table 2). When UPCs were classified by type, relatively large numbers of PBDs were observed in the subjects with T6 injures. In contrast, although quite small in comparison with T6 injuries, ABDs and MTs were observed frequently in the subjects with T12 injuries.

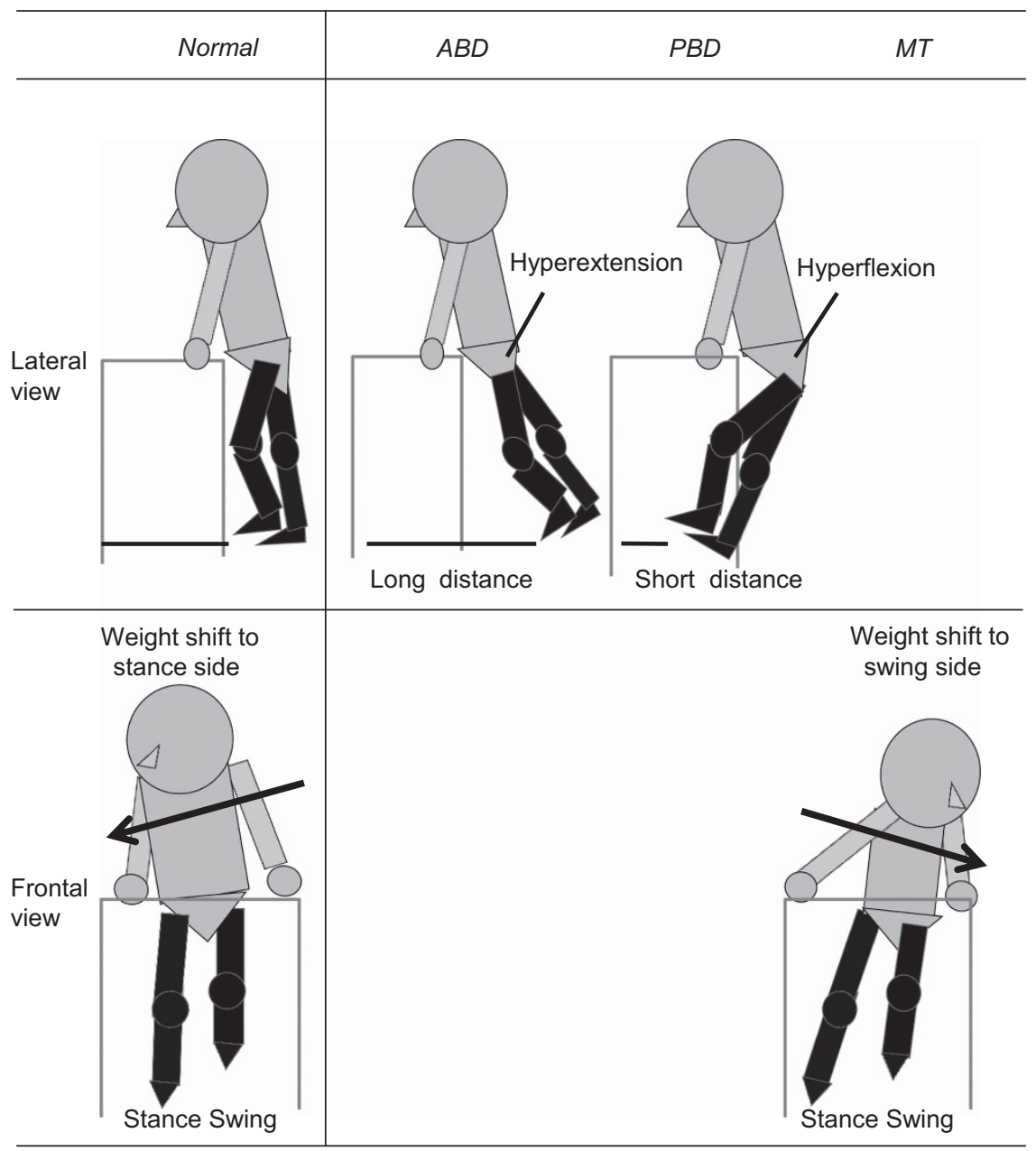

Figure 2 Representation of the type of unexpected postural changes (UPCs). Anterior breakdown (ABD) was defined as when both hip joints become hyperextended because of the long distance between the subjects and the walker. Posterior breakdown (PBD) was defined as when both hip joints become hyperflexed because of the short distance between the subjects and the walker. Mal-timing (MT) was defined as when the timing of the weight shift in subjects was different from the timing of the gait cycle of the WPAL. 
Table 2 Frequency of unexpected postural changes

\begin{tabular}{|c|c|c|c|c|c|}
\hline & Subject & Total trial time (h) & Walking time (h) & Total (incidents) & Total (incidents/h) \\
\hline \multirow{2}{*}{ T6 } & B & 28.5 & 20.0 & 20 & 1.00 \\
\hline & Mean (s.d.) & $25.00(6.06)$ & $17.67(4.04)$ & $24.00(8.72)$ & $1.36(0.35)$ \\
\hline \multirow[t]{2}{*}{$T 12$} & D & 19.5 & 14.0 & 2 & 0.14 \\
\hline & $\mathrm{E}$ & 27.0 & 19.0 & 11 & 0.58 \\
\hline
\end{tabular}

Table 3 Frequency of each type of unexpected postural change

\begin{tabular}{lllll}
\hline & Subject & \multicolumn{1}{c}{$\begin{array}{c}A B D \\
\text { (incidents/h) }\end{array}$} & $\begin{array}{c}\text { PBD } \\
\text { (incidents/h) }\end{array}$ & MT (incidents/h) \\
\hline T6 & A & 0.24 & 1.11 & 0.08 \\
& B & 0.10 & 0.60 & 0.30 \\
& C & 0.00 & 1.45 & 0.25 \\
& Mean (s.d.) & $0.11(0.12)$ & $1.06(0.43)$ & $0.21(0.12)$ \\
T12 & D & 0.00 & 0.00 & 0.15 \\
& E & 0.32 & 0.05 & 0.21 \\
& Mean (s.d.) & $0.16(0.22)$ & $0.03(0.04)$ & $0.18(0.05)$ \\
Total & Mean (s.d.) & $0.13(0.14)$ & $0.64(0.64)$ & $0.20(0.09)$ \\
\hline
\end{tabular}

Abbreviations: $\mathrm{ABD}$, anterior breakdown; MT, mal-timing; $\mathrm{PBD}$, posterior breakdown.

\section{DISCUSSION}

The present study analyzed the occurrence of UPCs during gait training with a WPAL in five SCI patients. UPCs were observed 85 times from gait training videos over a period of 2 years. UPCs could be classified into three types (PBDs, ABDs and MTs). The incidence of UPCs was $0.96 \pm 0.62$ (incidents/h/subject). PBDs were the most frequently observed UPCs. The time-course data of UPCs showed a gradual decrease in their incidence. For the neurological level-specific differences, UPCs were observed more frequently in patients with T6 injuries than in those with T12 injuries. These findings are critical observational evidence for improving the efficacy and safety of robotassisted gait training in SCI patients.

With regard to PBDs, their prevention is particularly important because a posterior fall as a result of a PBD incurs the highest risk of a femoral fracture. ${ }^{12}$ In addition, because bone density after an SCI tends to decrease, ${ }^{13}$ the possibility of a femoral fracture might be increased further. PBDs might be caused by a temporal delay of moving the walker forward. This delay is the determining factor in a near collision between the trunk of the user and the walker, and consequently makes it difficult for the user to move their trunk over an anterior stance limb.

For ABDs, the present results showed that they were the result of reduced toe clearance during the swing phase and following contact between the distal foot of the WPAL and the floor surface. It might be caused by anteversion of the lower leg in the stance phase, resulting from excessive extension of the hip joint. As almost all users can recover their posture from an $\mathrm{ABD}$ by pushing up using the walker, it might not be as serious as a PBD.

MTs were observed when the timing of the weight shift in the subjects was different from the timing of the gait cycle of the WPAL. When MTs occurred, the trunk was moved posteroinferiorly. When a single MT occurs, SCI patients can recover to a stable gait

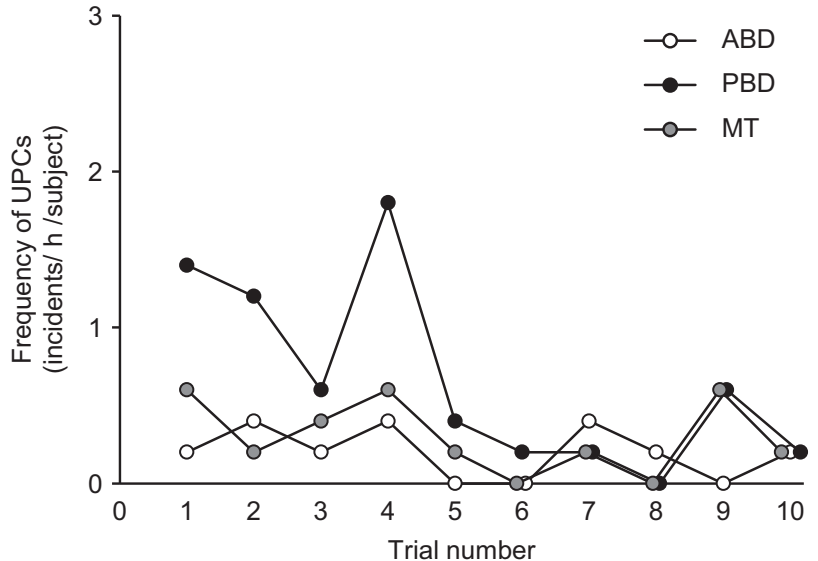

Figure 3 Time-course data of each type of unexpected postural change (UPC). Time courses of $\operatorname{ABD}(O), \operatorname{PBD}(\bigcirc)$ and $M T(\odot)$.

position by pushing up using the walker. However, it is possible that consecutive multiple MTs could lead to a PBD. Therefore, it is necessary to carry out sufficient practice to achieve the gait-assisted rhythm of the WPAL before starting independent WPAL walking with the walker. A previous study also reported that it is necessary to perform sufficient gait training before progressing to robot-assisted gait. $^{14}$

For 2 years, the average rate of the incidence of UPCs was $0.96 \pm 0.62$ (incidents/h/subject). Until now, there is no study on the rate of fall frequency during robot-assisted gait training in SCI patients. In patients with subacute stroke, previous studies reported that the rate of fall frequency ranged from 0.0002 to 0.0007 (incidents/ $\mathrm{h} /$ subject) during the hospital stay period including training session. ${ }^{15-17}$ In addition, the most frequent location was not the exercise room but rather the patient's room $\left(71.4 \%,{ }^{15} 63 \%{ }^{16}\right.$ and $51 \%$ 17 as a percentage of the total, respectively). Although these are not directly comparable, the rate of frequency of UPCs during robotassisted gait training in SCI patients might be relatively high in a variety of settings for inpatient rehabilitation. Thus, it has to be administered with particular care.

For the time-dependent changes in the occurrence of UPCs, the most frequently observed UPCs were PBDs in the early training period. By repetitive training, the frequency of UPCs decreased. As stated above, gait using WPAL was combined with a walker. Thus, the achievement of independent gait with WPAL requires training for lateral weight shifts, as well as backward and forward weight shifts, including control of the walker. The acquisition of motor skills such as gait needs feedback; ${ }^{18}$ however, complete SCI patients 
(American Spinal Injury Association classification A) lose somatosensory input from the limbs below the level of the lesion. Thus, it is difficult for these patients to receive feedback of the location of their lower limbs during gait. When they keep walking without realizing that the distance between the body of the WPAL and the walker has changed, UPCs might occur. Therefore, the decrease in the frequency of all UPCs with the progress of gait training might be caused by an improvement of walker manipulation, movement of the center of gravity and the ability to predict lower leg position from residual sensory information, for example, from the trunk. In fact, with repetitive practice, all patients could push the stop button on the walker to halt WPAL motion following the improvement of their ability to predict UPCs. Thus, the ability to detect postural changes based on residual sensory feedback during gait is critically important in wearable robot-assisted gait.

However, the frequencies of $\mathrm{ABD}$ and MT were not significantly improved. The results suggest that there are two possibilities. First, a floor effect might limit the improvement because both UPCs were rarely observed from the beginning of gait training. Second, each UPC might have specific factors to interfere with the decrease in the frequency. $\mathrm{ABD}$ is caused by the result of reduced toe clearance during the swing phase and following contact between the distal foot of the WPAL and the floor surface. When the contact occurred, it would immediately and seriously affect the upright posture. On the other hand, MT is caused by out of synchronization between the timing of weight shift in the subjects and the gait cycle of the WPAL. In healthy subjects, an increase in attentional demand decreases walking stability. ${ }^{19}$ In addition, for paraplegic patients, walking requires more attentional demand than for healthy subjects. ${ }^{20}$ When some high attentional demand tasks (e.g. curving road or talking to someone) occur, they would contribute to the occurrence of MT. Thus, even if the user achieves independent walking with WPAL, it might be difficult to completely avoid ABD or MT.

There was a difference in the frequency of observed UPCs between T6 and T12 injuries. UPCs were observed more frequently in patients with T6 injuries than in those with T12 injuries. PBDs occurred frequently in patients with T6 injuries, whereas ABDs and MTs occurred frequently in those with T12 injuries, even though the total frequency of UPCs was low in T12 patients. These results suggest that the difference of UPC type might be associated with the difference of residual posture control ability between patients with T6 and T12 injuries. When T6 and T12 injuries are compared, the function of the musculus erector spinae is different. The musculus erector spinae is an important compensatory mechanism to prevent falling when SCI patients are in the upright posture. ${ }^{21}$ The results of the present study suggest that the compensatory mechanism using this muscle might work particularly well in the PBDs. From the perspective of what is efficacious training for $\mathrm{T} 6$ and T12, the results suggest that there is not much difference between both levels, because the difference of residual functions in T6 and T12 does not affect presumed mechanisms of improvement (an improvement of walker manipulation, movement of the center of gravity and the ability to predict lower leg position from residual sensory information).

In summary of discussion, UPCs during robot-assisted gait training could be classified into three types (PBDs, ABDs and MTs). PBDs, which were most frequent, might be caused by a temporal delay of moving the walker forward. ABDs might be caused by anteversion of the lower leg in the stance phase. MTs might be caused by the difference between the timing of the weight shift and the timing of the gait cycle of the WPAL. With enough practice, UPCs showed a gradual decrease. For the neurological level-specific differences, UPCs were observed more frequently in patients with T6 injuries than in those with T12 injuries, because of difference from residual function of musculus erector spinae.

\section{Limitations}

There are some limitations of this study that merit consideration. Because the WPAL system has unique structure characterized by medial hip joint and no pelvic or trunk support, most of the other exoskeleton devices, as typified by Rewalk system, ${ }^{22,23}$ might show different trends. These devices contain bilateral hip and knee joint motors, rigid pelvic and trunk support, and require users to continue to lean forward while simultaneously bringing both crutches to the front to a standing balanced position. ${ }^{24}$ This structure and posture might increase the ABD and decrease the PBD. Although previous study reported occasional loss of balance during walking with Rewalk, ${ }^{23}$ it was not described in any detail concerning the type of UPCs. The small number of SCI patients might limit the strength of our results. In particular, the comparison between T6 and T12 injuries requires further study with additional subjects because each group has only a couple of subjects, which makes the results weaker. In addition, as all subjects in this study had lower thoracic SCIs, further investigations are needed to determine the incidence of UPCs in patients with cervical and upper thoracic SCIs.

\section{CONCLUSION}

The present study investigated the type and incidence of UPCs during robot-assisted gait training in SCI patients. PBD was the most commonly observed UPC during gait with a wearable robot. Moreover, it was prominent during the early stages of gait training with the walker without the harness and in patients with a relatively high level of injury. It is generally acknowledged that robot-assisted gait needs to be secured safely when used in clinical practice. ${ }^{25}$ The significance of this study is that it provides the first observational evidence with regard to UPCs during robot-assisted gait training in SCI patients. These findings would be useful for the development of an effective training program.

\section{DATA ARCHIVING}

There were no data to deposit.

\section{CONFLICT OF INTEREST}

TT is a salaried employee of the ASKA Corporation, which produces WPAL. $\mathrm{AU}$ is a salaried employee of the Tomei Brace Company, which distributes WPAL. The remaining authors declare no conflict of interest.

\section{ACKNOWLEDGEMENTS}

Our heartfelt appreciation goes to the late Professor Toru Suzuki who provided helpful advice on experimental design and suggestions on the interpretation of present results. We thank also Hagemu Higuchi and Kiyokazu Nakamura for their helpful data collection and technical assistance. We also thank all subjects for participating in the study. This work is financially supported by the NEDO grant for Basic Technology Development for Practical Application of Human Support Robots (8068149) and Practical Development of Industrial Technology (8080694), and fund from Aichi Science and Technology Foundation (22-2).

1 Maynard FM Jr, Bracken MB, Creasey G, Ditunno JF Jr, Donovan WH, Ducker TB et al. International standards for neurological and functional classification of spinal cord injury. American Spinal Injury Association. Spinal Cord 1997; 35: 266-274. 
2 Harvey LA, Adams R, Chu J, Batty J, Barratt DA. Comparison of patients' and physiotherapists' expectations about walking post spinal cord injury: a longitudinal cohort study. Spinal Cord 2012; 50: 548-552.

3 Morganti B, Scivoletto G, Ditunno P, Ditunno JF, Molinari M. Walking index for spinal cord injury (WISCI): criterion validation. Spinal Cord 2005; 43: 27-33.

4 Battaglino RA, Lazzari AA, Garshick E, Morse LR. Spinal cord injury-induced osteoporosis: pathogenesis and emerging therapies. Curr Osteoporos Rep 2012; 10: 278-285.

5 Dijkers MP. Correlates of life satisfaction among persons with spinal cord injury. Arch Phys Med Rehabil 1999; 80: 867-876.

6 Fuhrer MJ, Rintala DH, Hart KA, Clearman R, Young ME. Relationship of life satisfaction to impairment, disability, and handicap among persons with spinal cord injury living in the community. Arch Phys Med Rehabil 1992; 73: 552-557.

7 Major RE, Stallard J, Rose GK. The dynamics of walking using the hip guidance orthosis (hgo) with crutches. Prosthet Orthot Int 1981; 5: 19-22.

8 Saitoh E, Suzuki T, Sonoda S, Fujitani J, Tomita Y, Chino N. Clinical experience with a new hip-knee-ankle-foot orthotic system using a medial single hip joint for paraplegic standing and walking. Am J Phys Med Rehabil 1996; 75: 198-203.

9 Arazpour M, Bani MA, Hutchins SW, Jones RK. The physiological cost index of walking with mechanical and powered gait orthosis in patients with spinal cord injury. Spinal Cord 2013; 51: 356-359.

10 Tanabe S, Hirano S, Saitoh E. Wearable Power-Assist Locomotor (WPAL) for supporting upright walking in persons with paraplegia. NeuroRehabilitation 2013; 33 . 99-106.

11 Tanabe S, Saitoh E, Hirano S, Katoh M, Takemitsu T, Uno A et al. Design of the Wearable Power-Assist Locomotor (WPAL) for paraplegic gait reconstruction. Disabil Rehabil Assist Technol 2013; 8: 84-91.

12 Nankaku M, Kanzaki H, Tsuboyama T, Nakamura T. Evaluation of hip fracture risk in relation to fall direction. Osteoporos Int 2005; 16: 1315-1320.

13 Dudley-Javoroski S, Shields RK. Dose estimation and surveillance of mechanical loading interventions for bone loss after spinal cord injury. Phys Ther 2008; 88 : 387-396.
14 Reinkensmeyer DJ, Aoyagi D, Emken JL, Galvez JA, Ichinose W, Kerdanyan G et al. Tools for understanding and optimizing robotic gait training. J Rehabil Res Dev 2006; 43: 657-670.

15 Sze KH, Wong E, Leung HY, Woo J. Falls among Chinese stroke patients during rehabilitation. Arch Phys Med Rehabil 2001; 82: 1219-1225.

16 Nyberg L, Gustafson Y. Patient falls in stroke rehabilitation. A challenge to rehabilitation strategies. Stroke 1995; 26: 838-842.

17 Tutuarima JA, van der Meulen JH, de Haan RJ, van Straten A, Limburg M. Risk factors for falls of hospitalized stroke patients. Stroke 1997; 28: 297-301.

18 Tate JJ, Milner CE. Real-time kinematic, temporospatial, and kinetic biofeedback during gait retraining in patients: a systematic review. Phys Ther 2010; 90: 1123-1134.

19 Lajoie Y, Teasdale N, Bard C, Fleury M. Attentional demands for static and dynamic equilibrium. Exp Brain Res 1993; 97: 139-144.

20 Lajoie Y, Barbeau H, Hamelin M. Attentional requirements of walking in spinal cord injured patients compared to normal subjects. Spinal Cord 1999; 37: 245-250.

21 Lin KH, Lu TW, Hsu PP, Yu SM, Liao WS. Postural responses during falling with rapid reach-and-grasp balance reaction in patients with motor complete paraplegia. Spinal Cord 2008; 46: 204-209.

22 Zeilig G, Weingarden H, Zwecker M, Dudkiewicz I, Bloch A, Esquenazi A. Safety and tolerance of the ReWalk ${ }^{\mathrm{TM}}$ exoskeleton suit for ambulation by people with complete spinal cord injury: A pilot study. J Spinal Cord Med 2012; 35: 96-101.

23 Esquenazi A, Talaty M, Packel A, Saulino M. The ReWalk powered exoskeleton to restore ambulatory function to individuals with thoracic-level motor-complete spinal cord injury. Am J Phys Med Rehabil 2012; 91: 911-921.

24 Spungen AM, Asselin P, Fineberg DB, Kornfeld SD, Harel NY. Exoskeletal-assisted walking for persons with motor-complete paraplegia. Research and Technology Organization, Human Factors, and Medicine Panel: North Atlantic Treaty Organization 2013. Available from: http://www.cso.nato.int

25 Galvez JA, Reinkensmeyer DJ. Robotics for gait training after spinal cord injury. Top Spinal Cord Inj Rehabil 2005; 11: 18-33. 